34.P11

\title{
Graft-Host Glutamatergic Neuronal Interaction in the Neocortex
}

\author{
*V.V. Senatorov, I. Vilagi, I. Tarnawa, I. Banczerowski-Pelyhe and Z. Fulop \\ "Institute for Experimental Medicine, St. Petersburg, Russia, 197022; \\ ${ }^{*}$ Loeb Research Institute, Ottawa Civic Hospital, Ottawa, Canada, K1Y 4E9; \\ Department of Comparative Physiology of Eotvos L. University, Budapest, Hungary
}

Recent evidence indicates that grafts of fetal neocortical tissue develop reciprocal connections with surrounding neocortical tissue of the host, but the nature of the neuromediator in these connections is still unclear. Excitatory synaptic transmission in the mammalian neocortex activated by glutamate is mediated by both N-methyl-D-aspartate (NMDA) and nonNMDA receptors. The non-NMDA receptorchannel complex mediates a fast voltageindependent synaptic conductance, while the NMDA receptor-channel complex mediates a slow, voltage-dependent conductance, governed by magnesium ion $\left(\mathrm{Mg}^{2+}\right)$ block. To conduct ionic current efficiently when activated by glutamate, the NMDA channel must be depolarized to voltage range whereby the $\mathrm{Mg}^{2+}$ block is removed from the NMDA channels. In our present study conducted in perfused rat neocortical slices we utilized low extracellular magnesium to study the NMDA receptormediated spontaneous and evoked field potentials (FPs) recorded from host and graft tissues in order to investigate host/graft interconnections of glutamatergic neurons.

Fetal (E15) neocortical grafts were transplanted into the lesion cavity of adult neocortex 1-3 months before electrophysiological experiments. Seventeen slices containing graft tissue were obtained from 17 host-rats. Parallel extracellular recordings were made through two glass micropipettes (filled with $4 \mathrm{M} \mathrm{NaCl}, 10-15 \mathrm{M} \Omega$ ) simultaneously from both graft and host parts of the slice in standard and $\mathrm{Mg}^{2+}$-free artificial cerebrospinal fluid (ACSF). The host and graft tissues were stimulated alternately in order to elicit evoked FPs from both sites. A single electrical shock (0-.1 ms, 2-7 V) was given at 0.1-1.0 $\mathrm{Hz}$. After the recordings, neurofilament poly- peptide $(160 \mathrm{kDa})$ immunostaining was carried out to visualize neural fibers in the slice.

In standard ACSF, electrical stimulation of the host elicited evoked FPs in 4/17 grafts. However in $\mathrm{Mg}^{2+}$-free ACSF, the same stimulation initiated evoked FPs in an additional 6 grafts and significantly $(P<0.01)$ increased the mean amplitude and duration of the evoked FPs. In addition, stimulation of 5 grafts elicited evoked FPs in the host tissue in $4 / 5$ slices perfused in standard ACSF, suggesting reciprocal functional interaction of graft and host tissues. The amplitude and duration of the evoked FPs recorded in host tissue were also significantly $(\mathrm{P}<0.01)$ enhanced in $\mathrm{Mg}^{2+}$-free ACSF (5/5 slices).

During perfusion of $\mathrm{Mg}^{2+}$-free ACSF, spontaneous epileptiform FPs were recorded throughout different parts of the slice. Simultaneous extracellular recordings from graft and host tissues demonstrated that these spontaneous epileptiform FPs burst synchronously in graft and host parts of 10/17 slices. Furthermore, bath application of the glutamate agonist NMDA $(10 \mu \mathrm{M})$ enhanced the frequency of occurrence of spontaneous epileptiform FPs in both graft and host parts of all tested slices and this response mimicked evoked FPs elicited by electrical stimulation. Application of the selective competitive NMDA-antagonist APV (20 $\mu \mathrm{M})$ and the non-competitive NMDA-antagonist ketamine $(20 \mu \mathrm{M})$ inhibited spontaneous epileptiform FPs and decreased the amplitude of evoked FPs, suggesting that the NMDA receptor was activated by glutamate which was released from stimulated fibers.

Post-recording immunostaining for neurofilament revealed the presence of networks of neural fibers within the graft. In 10/17 slices where physiological graft/host connections were 
found, immunopositive fibers in the graft were also shown to cross the border to the surrounding host tissue, thus creating a common neuropile.

Our data demonstrated that: 1) host and graft have reciprocal connections which can be activated in the absence of extracellular $\mathrm{Mg}^{2+} ; 2$ ) $\mathrm{Mg}^{2+}$-free medium initiated spontaneous epileptiform FPs which burst synchronously in graft and host tissues, and are sensitive to NMDA and blocked by its antagonists; 3) NMDA application can mimic evoked FPs in the graft elicited by electrical stimulation of the host; 4) these evoked FPs can be inhibited by NMDA antagonists. Thus, the NMDA activated receptor-channel complex participates in mediating graft-host glutamatergic neuronal interaction in the neocortex. The glutamatergic neurons from grafted neocortical tissue can be functionally integrated into circuits within the neocortex. NMDA channels play a major role in the host-graft interaction suggesting their critical link in the mechanisms involved in the development of plasticity in neural tissues. When neurons are depolarized sufficiently to relieve magnesium blockade of NMDA channels, $\mathrm{Ca}^{2+}$ flows through the channel and acts as a second messenger to activate a variety of $\mathrm{Ca}^{2+}$. dependent biochemical cascades which ultimately affect neurite outgrowth and synaptic connectivity. 

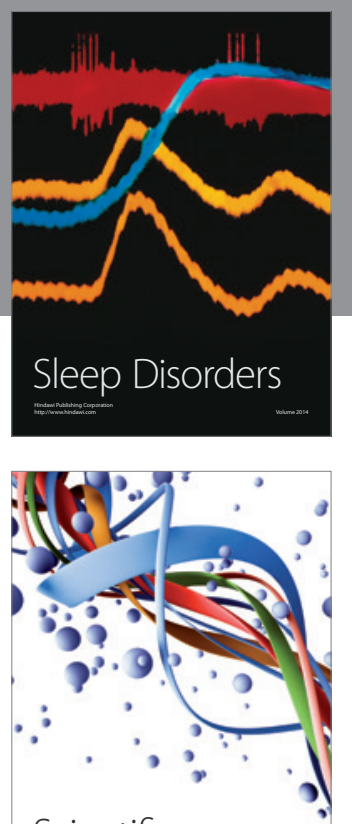

Scientifica
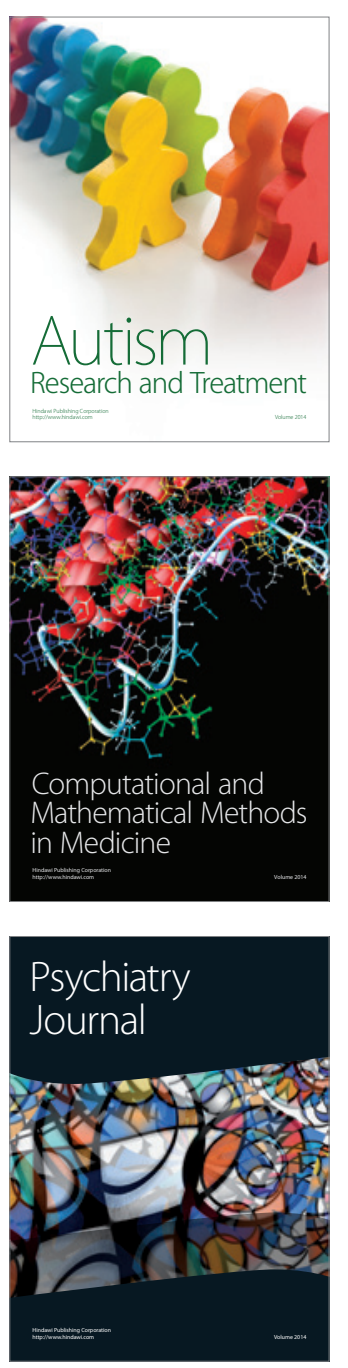
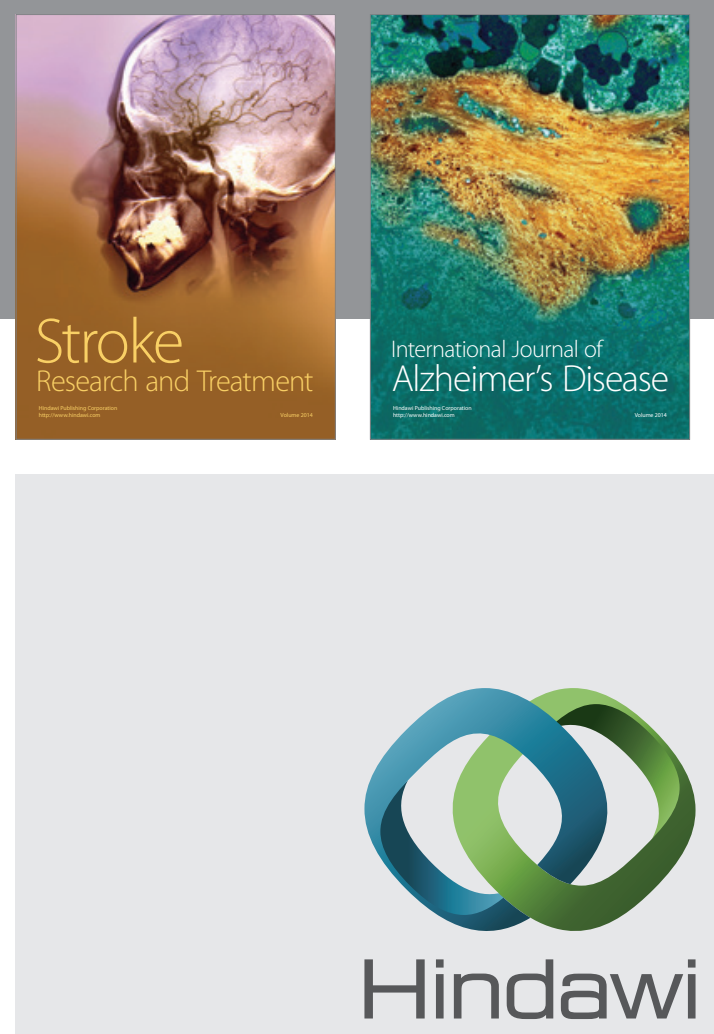

Submit your manuscripts at

http://www.hindawi.com
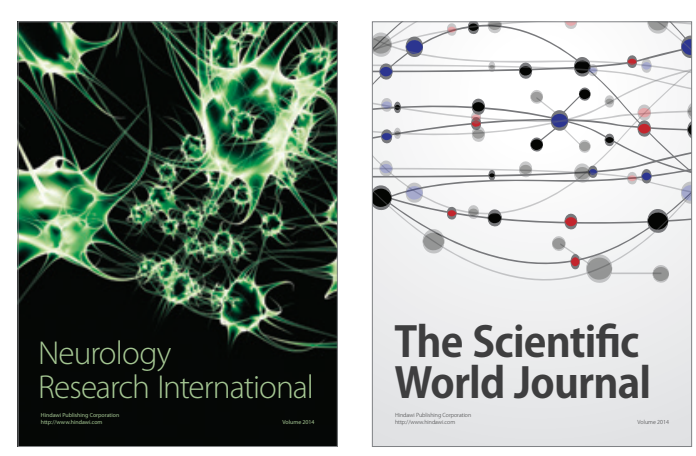

The Scientific World Journal

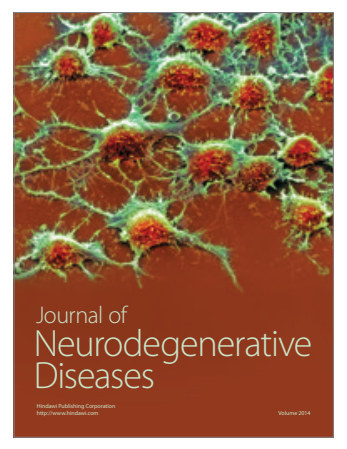

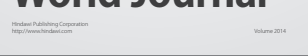

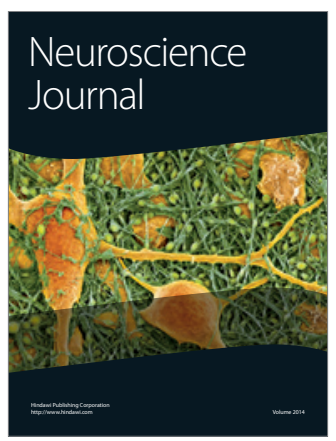

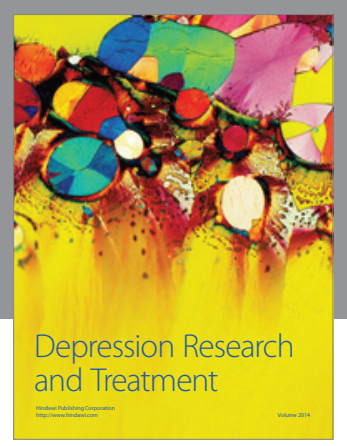
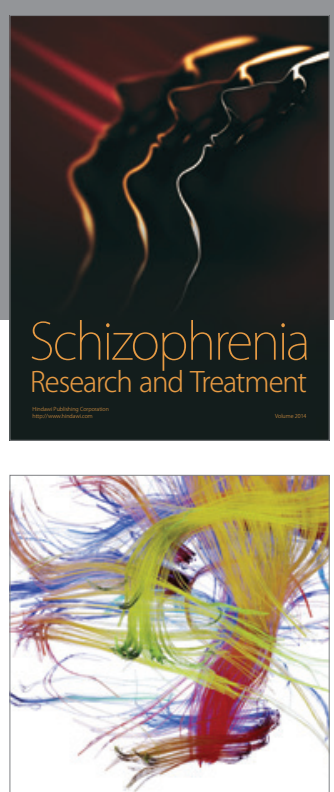

Brain Science

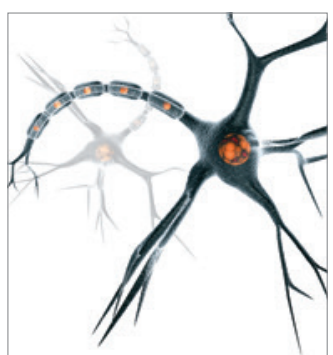

Neural Plasticity
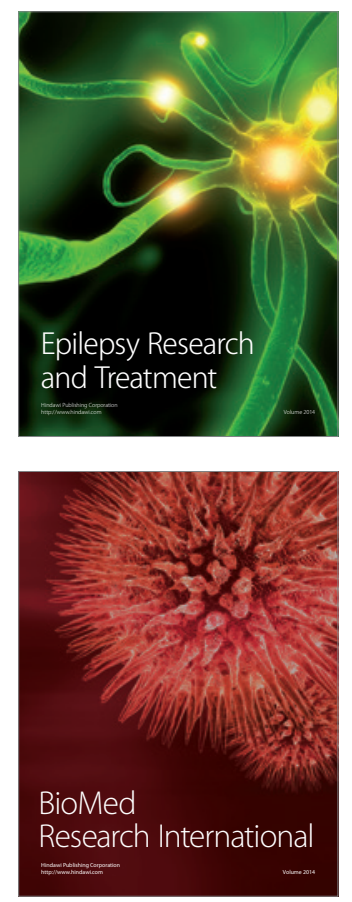

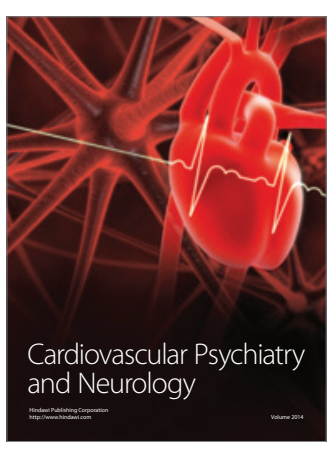

Parkinson's

Disease
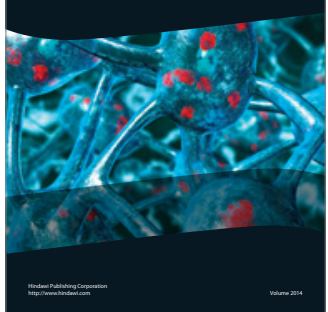